\title{
STUDIES OF THE SPECIFICITY OF UREAPLASMAS FOR MARMOSETS
}

\author{
Patricia M. Furr, C. M. Hetherington and D. Taylor-Robinson \\ Divisions of Communicable Diseases and Comparative Medicine, \\ Clinical Research Centre, Watford Road, Harrow, Middlesex HAI $3 U \mathrm{UJ}$
}

\begin{abstract}
UREAPLASMAS have been isolated from several animal species, including the marmoset (Callithrix jacchus) (Furr, Taylor-Robinson and Hetherington, 1976). However, strains of ureaplasma isolated from a single species of animal exhibit as much antigenic heterogeneity, in serological tests, as do strains isolated from different species of animals (Taylor-Robinson and Furr, 1973). This makes it difficult to determine the animal origin of strains if they cannot be identified with known antisera, although differences have recently been detected in the guanine-cytosine ratio of ureaplasmas from different animals (Howard and Gourlay, personal communication). There is little information on the species specificity of ureaplasmas, and the present study was undertaken to determine whether host specificity might assist in classification.
\end{abstract}

\section{MATERIALS AND METHODS}

Ureaplasmas and culture methods. The ureaplasmas included the following strains: two bovine strains, $417 / 1$ and MY9083, isolated from the respiratory tract and subcultured six times and once, respectively, and a further bovine strain, SP1637, isolated from the prepuce and subcultured twice; a canine strain, SP1701, isolated from the male urethra and subcultured seven times, and a fresh canine isolate, MY9308, from the vagina; a squirrel monkey strain, SP1625, isolated from the throat and subcultured seven times; a human strain, $43 \alpha$, isolated from the male urethra and subcultured six times; two fresh marmoset isolates, MY9347 and MY9266, isolated from the vagina and throat, respectively. These strains were grown, for the inoculation of marmosets, in urea-containing liquid medium (Taylor-Robinson et al., 1971) without thallium acetate. Subsequently, re-isolation of the strains from the marmosets was attempted, with oral and genital swabs taken daily for a week and then weekly. The swabs were expressed into $1.8 \mathrm{ml}$ of medium and serial 10-fold dilutions were made and incubated at $37^{\circ} \mathrm{C}$; infectivity was expressed, in colour-changing units (c.c.u.), as the highest dilution that produced a colour change of the medium from yellow to magenta (Furr et al., 1976).

Preparation and inoculation of marmosets. Adult marmosets, bred at the Clinical Research Centre (Furr et al., 1976), were given a 5-day course of minocycline (Minocin, Lederle) to eliminate endogenous marmoset ureaplasmas; the drug was placed in the drinking water at a concentration of $0.3 \mathrm{mg}$ per $\mathrm{ml}$. Earlier experiments had shown that marmosets were readily cleared of their normal flora of ureaplasmas by such treatment and that there was no reappearance of the organisms, under normal caging conditions, over an observation period of 40 days. The antibiotic course was discontinued several days before inoculation with ureaplasmas, to avoid interference by residual amounts of minocycline. Oral and genital swabs were taken just before inoculation to confirm the absence of endogenous ureaplasmas. Antibody tests (vide infra) showed that none of the 19 marmosets used in the experiments possessed detectable pre-existing antibody against bovine, canine, squirrel-monkey or human

Received 20 Mar. 1978; accepted 27 Apr. 1978.

J. MED. MICROBIOL.-VOL. 11 (1978) 
ureaplasmas. Only two of them had antibody to marmoset ureaplasmas; one, challenged with a bovine ureaplasma strain, had an antibody titre of 8 , and the other, challenged with a human strain, had a titre of 64 .

The marmosets, unsedated, were inoculated orally by allowing $0.5 \mathrm{ml}$ of ureaplasma culture to run slowly into the throat from a tuberculin syringe, and genitally either by intro-

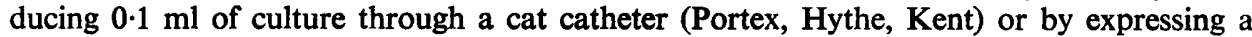
swab, soaked in PPLO broth containing ureaplasmas, in the vagina or under the prepuce.

Tests for antibody. Metabolism-inhibition antibody titres were measured by a microtechnique (Purcell et al., 1966) in growth medium containing guinea-pig serum $1 \%(\mathrm{v} / \mathrm{v})$.

\section{RESULTS}

\section{Attempts to infect marmosets with ureaplasmas from other animal species}

Bovine ureaplasmas. The two bovine respiratory strains, 417/1 and MY9083, were administered both orally and genitally, in a dose of $2.5 \times 10^{7}$ c.c.u., to two female marmosets and to three female and one male marmoset, respectively. The bovine genital strain, SP1647, was administered both orally and genitally, in a dose of $2.5 \times 10^{6}$ c.c.u., to two male marmosets. In none of the eight marmosets did infection occur.

Five of these marmosets were subsequently challenged with marmoset ureaplasmas; the genital strain, MY9347, was administered both orally and genitally, in a dose of $2.5 \times 10^{5}$ c.c.u., to three of them, one male and two female, and the respiratory strain, MY9266, in a dose of $2.5 \times 10^{4}$ c.c.u., to two females. In all five marmosets, oral infection was detected within 1-7 days and persisted for observation periods of 8-36 days; maximal infectivity titres in the mouth ranged from $5 \times 10^{4}$ to $5 \times 10^{7}$ c.c.u. per ml. On the other hand, neither of the marmoset strains used for challenge established itself in the genital tract of any of the five marmosets. However, when three of the animals were again challenged genitally, this time by the direct transfer of marmoset ureaplasmas $\left(2.5 \times 10^{6}\right.$ c.c.u. $)$ from their own mouths by means of swabs, infection lasting 7 days or more occurred.

Canine ureaplasmas. Two genital strains, SP1701 $\left(2.5 \times 10^{5}\right.$ c.c.u. $)$ and MY9308 $\left(2.5 \times 10^{7}\right.$ c.c.u. $)$, were each administered both orally and genitally to two male marmosets and to one male and one female marmoset, respectively. Neither strain produced an infection. However, when one of the marmosets was caged with another carrying naturally acquired marmoset ureaplasmas in the mouth, it became infected, in the mouth, within 4 days.

Simian ureaplasmas. A squirrel-monkey ureaplasma strain, SP1625 $\left(2.5 \times 10^{7}\right.$ c.c.u. $)$, was administered both orally and genitally to two female marmosets. A persistent infection, lasting 35 days or more and achieving maximal infectivity titres of $5 \times 10^{5}$ c.c.u. per $\mathrm{ml}$, was established in the throat of one animal, but in neither marmoset did genital infection occur. Unfortunately, an antiserum to the squirrel-monkey ureaplasma was not available, but the oral isolates could not be identified serologically as marmoset ureaplasmas. Two further attempts to infect the refractory marmoset were unsuccessful, but when it was caged with a naturally infected marmoset it became infected, in the mouth, with marmoset ureaplasmas within 3 days, achieving an infectivity titre of $5 \times 10^{4}$ c.c.u. per ml. 
Human ureaplasmas. Two of the female marmosets that had resisted infection with bovine ureaplasmas were challenged orally and genitally with $2.5 \times 10^{7}$ c.c.u. of the human strain, $43 \alpha$. Both animals became infected in the mouth and the genital tract, and organisms were detected, in both sites, within 1-2 days. They persisted in the mouth of one marmoset for 22-28 days and in the mouth of the other for 80 days, reaching maximal infectivity titres of $5 \times 10^{4}$ c.c.u. per $\mathrm{ml}$, but they persisted in the genital tracts for only about 2 weeks. The isolates from both marmosets were identified serologically as human ureaplasmas. When, after 80 days, human ureaplasmas could no longer be recovered from the second marmoset, it was housed with a marmoset naturally infected with marmoset ureaplasmas; it became infected with these ureaplasmas, in the mouth, within 6 days.

\section{Discussion}

Marmosets could not be infected even with large numbers of bovine or canine ureaplasmas, although the same animals were readily susceptible to experimental infection with human ureaplasmas and to experimental or natural infection with marmoset strains. Other animals, too, show evidence of host specificity for ureaplasmas. Thus, bovine ureaplasmas did not infect the genital tract of dogs (Taylor-Robinson and Furr, unpublished), and human ureaplasmas failed to infect the genital tract of goats (Gourlay, Howard and Taylor-Robinson, unpublished). On the other hand, both human and bovine ureaplasmas infected the mouse mammary gland (Howard et al., 1975), and human ureaplasmas infected the udder of the goat (Gourlay, Brownlie and Howard, 1973), but not of the cow (Howard, Gourlay and Brownlie, 1973). Organisms are likely to be trapped in the tissues of the mammary gland and this probably increases the likelihood of infection; on a mucosal surface ureaplasma receptors (Taylor-Robinson et al., 1971; Black, 1973) may be more important. However, nothing is as yet known of receptors on marmoset epithelial cells that could account for differences in susceptibility to infection by marmoset, human, bovine and canine ureaplasmas. Although the body temperature of the marmoset, which varies between $36^{\circ} \mathrm{C}$ in the dark and $39^{\circ} \mathrm{C}$ in the light (Hetherington, 1978), might influence susceptibility, this does not seem likely. The higher temperature should, in fact, be less likely to inhibit the growth of bovine and canine ureaplasmas than of human ureaplasmas, as the average rectal temperatures of cattle $\left(38.6^{\circ} \mathrm{C}\right)$ and dogs $\left(38.3^{\circ} \mathrm{C}\right)$ are higher than that of man. Immunity is likely to have an important influence on reinfection, but the absence of pre-existing serum antibody in the marmosets to bovine and canine ureaplasmas precludes this possibility in the present experiments.

Our results show that although marmosets exhibit host specificity for ureaplasmas, this specificity is incomplete. Marmosets could be infected experimentally with squirrel-monkey ureaplasmas and with those of human origin, although the genital tract was less susceptible than the throat; this is in accord with the less frequent presence of naturally occurring ureaplasmas in the genital tract of marmosets than in the throat (Furr et al., 1976). 
On the basis of marmoset susceptibility, it seems that human, marmoset, and squirrel-monkey ureaplasmas comprise a subgroup, separate from bovine and canine ureaplasmas. We are not suggesting that host specificity can be used as a strict means of classifying ureaplasmas, but such biological differences may be of value and deserve further study.

\section{SUMMARY}

Marmosets, from which endogenous ureaplasmas had been eradicated by treatment with minocycline, were tested for susceptibility to infection by ureaplasmas from the genital and respiratory tracts of other animal species. They could be infected with ureaplasmas of human and simian origin, but were resistant to bovine and canine ureaplasmas. The results indicated that human, marmoset and squirrel-monkey ureaplasmas may form a biological subgroup, distinct from bovine and canine ureaplasmas, and that host range should not be ignored as a parameter for classification.

\section{REFERENCES}

BlACK, F. T. 1973. Biological and physical properties of human T-mycoplasmas. Ann. N.Y. Acad. Sci., 225, 131.

FurR, P. M., TAYlor-Robinson, D. AND Hetherington, C. M. 1976. The occurrence of ureaplasmas in marmosets. Lab. Anims, 10, 393.

GourLAY, R. N., BROWNLIE, J. AND HowARD, C. J. 1973. Isolation of T-mycoplasmas from goats, and the production of subclinical mastitis in goats by the intramammary inoculation of human T-mycoplasmas. J. gen. Microbiol., 76, 251.

Hetherington, C. M. 1978. Circadian oscillations of body temperature in the marmoset Callithrix jacchus. Lab. Anims, 12, 104.

Howard, C. J., Anderson, J. C., Gourlay, R. N. AND TaYlor-Robinson, D. 1975. Production of mastitis in mice with human and bovine ureaplasmas (T-mycoplasmas). J. med. Microbiol., 8, 523.

Howard, C. J., GourLaY, R. N. AND BrownLIE, J. 1973. The virulence of T-mycoplasmas isolated from various animal species, assayed by intramammary inoculation in cattle. J. Hyg., Camb., 71, 163.

Purcell, R. H., Taylor-Robinson, D., Wong, D. and Chanock, R. M. 1966. Color test for the measurement of antibody to T-strain mycoplasmas. J. Bact., 92, 6.

TAYLOR-Robinson, D. AND FURR, P. M. 1973. The distribution of T-mycoplasmas within and among various animal species. Ann. N.Y. Acad. Sci., 225, 108.

Taylor-Robinson, D., Martin-Bourgon, C., Watanabe, T. and AdDey, J. P. 1971. Isolation of T-mycoplasmas from dogs and squirrel monkeys: biological and serological comparison with those isolated from man and cattle. J. gen. Microbiol., 68, 97. 